\title{
Pattern of Stroke and Short Term Outcome of COVID-19 Patients admitted in a Dedicated Stroke Unit at Referral Neurology Hospital of Bangladesh
}

\author{
Subir Chandra Das ${ }^{1}$, Rajib Nayan Chowdhury², Md. Moniruzzaman³, Mohammad Enayet Hussain 4 , \\ Quazi Deen Mohammad ${ }^{5}$, Md. Badrul Alam Mondal ${ }^{6}$, MS Jahirul Haque Chowdhury ${ }^{7}$ \\ ${ }^{1}$ Medical Officer, Department of Neurology, National Institute of Neurosciences \& Hospital, Dhaka, Bangladesh; ${ }^{2}$ Professor, \\ Department of Neurophysiology, National Institute of Neurosciences \& Hospital, Dhaka, Bangladesh; ${ }^{3}$ Medical Officer, Department \\ of Neurology, National Institute of Neurosciences \& Hospital, Dhaka, Bangladesh; ${ }^{4}$ Associate Professor, Department of \\ Neurology, National Institute of Neurosciences \& Hospital, Dhaka, Bangladesh; ${ }^{5}$ Professor of Neurology \& Director, \\ National Institute of Neurosciences \& Hospital, Dhaka, Bangladesh; ${ }^{6}$ Joint Director\&Professor of Neurology, National \\ Institute of Neurosciences \&Hospital, Dhaka, Bangladesh; ${ }^{7}$ Professor, Department of Clinical Neurology, National \\ Institute of Neurosciences \&Hospital,Dhaka, Bangladesh
}

[Received on: 22 November 2020; Accepted on: 12 December 2020; Published: 1 January 2021]

\begin{abstract}
Background: COVID-19 was declared as a pandemic by WHO on March 11, 2020. Though the most common and important presentation is with respiratory disease, reports of neurological features are increasing. Objective: In this study it was described the pattern of stroke in COVID-19 patients admitted in a dedicated stroke unit and their short term outcome. Methodology: This single arm, single centreprospective cohortstudy was conducted in the Stroke Unit of National Institute of Neurosciences and Hospital, Dhaka, Bangladesh from April to August, 2020 and wasincluded acute stroke patients irrespective of age and sex who were found RT-PCR positive for SARS CoV-2. The patientswere evaluated clinically, biochemically and by imaging. After diagnosis patients were referred to a COVIDdedicated hospital and were followed up over phone for 4 weeks from the day of admission in stroke unit to assess short term outcome. Results: Among the 41 patients, 21 were male and 20 female with an age range of 26 to 90 years where $58.5 \%$ patients were aged 60 and above. Majority (58.5\%) of them came from different urban areas. Common co-morbidities were Hypertension $(68.3 \%)$ and Diabetes mellitus (39\%). Most common presenting features were hemiplegia (48.8\%) and altered level of consciousness (39\%). More than seventy percent $(70.7 \%)$ patients had GCS of 10 or less and $56.1 \%$ patients had oxygen saturation $90 \%$ or less in room air. CT brain imaging revealed infarct in 39\% and hemorrhage in $51.2 \%$. Chest radiography revealed abnormality in $14.6 \%$ cases. Regarding the short term outcome, total $20(48.8 \%)$ patients died where 7 died before referral to COVID-19 dedicated hospital. Death was higher in hemorrhagic stroke but there was no significant association between outcome and the type of stroke ( $p$ value $>0.05$ ). Conclusions: Hemorrhagic stroke is the most commonly occurring stroke among the COVID-19 patients and associated with poor outcome. [Journal of National Institute of Neurosciences Bangladesh, January 2021;7(1): 25-28]
\end{abstract}

Keywords: SARS CoV-2; COVID-19;stroke;hemorrhagic stroke

Correspondence: Dr. Subir Chandra Das, Medical Officer, Department of Neurology, National Institute of Neurosciences \& Hospital, Dhaka, Bangladesh; Cell no.: +8801716450560; Email: drsubir12@gmail.com

Conflict of interest: There is no financial conflict of interest relevant to this paper to disclose.

Funding agency: This research project was not funded by any group or any institution.

Contribution to authors: Das SC, Chowdhury RN, Moniruzzaman M, Hussain ME contributed from the protocol preparation, data collection up to report writing. Manuscript writing was performed by Das SC; Mohammad QD, Alam MB, Chowdhury MSJH have revised the manuscript.

How to cite this article: Das SC, Chowdhury RN, Moniruzzaman M, Hussain ME, Mohammad QD, Alam MB, Chowdhury MSJH. Pattern of Stroke and Short Term Outcome of COVID-19 Patients admitted in a Dedicated Stroke Unit at Referral Neurology Hospital of Bangladesh. J Natl Inst Neurosci Bangladesh, 2021;7(1):25-28

Copyright: (C2021. Das et al. Published by Journal of National Institute of Neurosciences Bangladesh. This article is published under the Creative Commons CC BY-NC License (https://creativecommons.org/licenses/by-nc/4.0/). This license permits use, distribution and reproduction in any medium, provided the original work is properly cited, and is not used for commercial purposes.

\section{Introduction}

Corona virus disease 2019 (COVID-19) caused by SARSCov-2 virus, first emerged from Wuhan city, Hubei province of $\mathrm{China}^{1}$.The epidemic spread rapidly worldwide within 3 months and was declared as a pandemic by WHO on March 11, 2020 ${ }^{2}$.Severe acute respiratory illness with fever and respiratory symptoms, such as cough and shortness of breath, comprise the main clinical presentations. But unusual manifestations, such as patients without respiratory symptoms or only very mild symptoms are rising worldwide. Initially it was thought to involve primarily the respiratory system like other coronaviruses likesevere acute respiratory syndrome (SARS) and theMiddle East Respiratory 
Syndrome (MERS), later it wasfound to have involved other systems as well includingthe nervous system ${ }^{3-4}$.

Although the most common and important presentation is with respiratorydisease, reports of neurological features are increasing. These features appear to be a combination of nonspecific complications of systemic disease, the effects of direct viral infection, or inflammation of the nervous system and vasculature, which can be para-infectious or post-infectious ${ }^{5}$. In one national registry of 125 patients with COVID-19 and neurological or psychiatric disease reported over a 3 -week period, $62.0 \%$ patients had a cerebrovascular event: $46.0 \%$ ischemic strokes, $7.0 \%$ intracerebral haemorrhages ${ }^{6}$. As the epidemic is ongoing, the pattern of neurological manifestations of COVID-19 especially CNS features still not well known worldwide. Whether the neurological features are directly attributed to the virus or it is just an associated infection is also a big question. In this study it had been described the pattern of stroke in COVID-19 patients admitted in a dedicated stroke unit and their short termoutcome.

\section{Methodology}

This was a single arm, single centre prospective cohort studyconducted in Stroke Unit of National Institute of Neurosciences and hospital, Dhaka, Bangladesh from April to August, 2020. The study included acute stroke patients irrespective of age and sex who were found RT-PCR positive for SARS CoV-2. The sampling method was purposive. All patients were clinically evaluated by Glasgow Coma Scale (GCS) and theywere also evaluated by CT Brain imaging, Chest X-ray and routine hematology and blood biochemistry. After diagnosis patients were referred to a covid dedicated hospital and we followed up the patients over phone for 4 weeks from the day of admission in stroke unit to assessshort term outcome. All the data were collected in a semi structured questionnaire by study physicians and analyzed by SPSS version 22.Chi-Square test was done to see the association of outcome with types of stroke.

\section{Results}

Total 41 patients were enrolled in the study. Among them $21(51.2 \%)$ male and $20(48.8 \%)$ were female with a male to female ratio of 1.05:1. Their age ranging from 26 to 90 year where most $(26.8 \%)$ of the patients were in 60-69 age group and 58.5\% patients were aged 60 and above. Majority (58.5\%) of them came from different urban areas. Common co-morbidities were Hypertension (68.3\%), Diabetes mellitus (39\%) and chronic kidney disease (2.4\%) (Table 1).

Table 1: Demographic characteristics and co-morbidities of the patients

\begin{tabular}{lcc}
\hline Age Group & Frequency & Percent \\
\hline 20 to 29 Years & 3 & 7.3 \\
30 to 39 Years & 3 & 7.3 \\
40 to 49 Years & 3 & 7.3 \\
50 to 59 Years & 8 & 19.5 \\
60 to 69 Years & 11 & 26.8 \\
70 to 79 Years & 8 & 19.5 \\
80 to 89 Years & 2 & 4.9 \\
More than 90 Years & 3 & 7.3 \\
Gender & & \\
Male & 21 & 58.5 \\
Female & 20 & 48.8 \\
Residence & & \\
Urban 24 & 24 & 58.5 \\
Rural 17 & 17 & 41.5 \\
Co-morbidities & & \\
HTN 28 & 28 & 68.3 \\
DM 16 & 16 & 39.0 \\
CKD 1 & 1 & 2.4 \\
\hline
\end{tabular}

Common presenting features were hemiplegia (48.8\%), altered level of consciousness (39\%), headache $(12.2 \%)$ and convulsion $(7.3 \%)$. Clinical evaluation revealed GCS of 10 or less in $70.7 \%$ patients and oxygen saturation of $90 \%$ or less in $56.1 \%$ patients (Table 2).

Table 2: Clinical features of the patients

\begin{tabular}{lcc}
\hline Features & Frequency & Percent \\
\hline Presenting Complaints & & \\
Loss of consciousness & 16 & 39.0 \\
Hemiplegia & 20 & 48.8 \\
Aphasia & 2 & 4.9 \\
Headache & 5 & 12.2 \\
Convulsion & 3 & 7.3 \\
Confusion & 10 & 24.4 \\
Examination findings GCS & & \\
$<7$ & 14 & 34.1 \\
7 to 10 & 15 & 36.6 \\
$>10$ & 12 & 29.3 \\
SpO2 & & \\
60 to $80 \%$ & 6 & 14.6 \\
80 to $90 \%$ & 17 & 41.5 \\
$>90 \%$ & 18 & 43.9 \\
\hline
\end{tabular}

CT brain imaging revealed infarct in 39\% and hemorrhage in $51.2 \%$ cases. Chest radiography revealed abnormality in $14.6 \%$ cases (Table 3 ). 
Table 3: Investigation findings of the patients

\begin{tabular}{lcc}
\hline Investigations & Frequency & Percent \\
\hline HbA1C & 10 & \\
$<6.5 \%$ & 3 & 24.4 \\
$6.5-9 \%$ & 3 & 7.3 \\
$>9 \%$ & & 7.3 \\
Chest X-ray & 34 & \\
Normal & 3 & 82.9 \\
Unilateral inflammatory lesion & 3 & 7.3 \\
Bilateral Inflammatory lesion & & 7.3 \\
CT scan of Head & 16 & \\
Infarction & 10 & 39.0 \\
Intracerebral hemorrhage & & 24.4 \\
Intracerebral hemorrhage with & 3 & \\
ventricular extension & 2 & 7.3 \\
Intraventricular hemorrhage & 2 & 4.9 \\
Subarachnoid hemorrhage & 6 & 14.6 \\
Unremarkable & 4 & 9.8 \\
\hline
\end{tabular}

Regarding the short term outcome, total 20 (48.8\%) patients died where 7 died before referral to COVID-19 dedicated hospital.Death was relatively higher in cases of hemorrhagic stroke but association of outcome with types of stroke was not statistically significant ( $p$ value $>0.05$ ) (Table 4).

Table 4: Association of Outcome with Types of Stroke

\begin{tabular}{lccc}
\hline Stroke type & Recovered & Death (\%) & P value \\
\hline Ischemic stroke & $12(29.3 \%)$ & $8(19.5 \%)$ & \\
Hemorrhagic stroke & $5(12.2 \%)$ & $10(24.4 \%)$ & 0.211 \\
SAH & $4(9.7 \%)$ & $2(4.9 \%)$ & \\
Total & $\mathbf{2 1 ( 5 1 . 2 \% )}$ & $\mathbf{2 0}(\mathbf{4 8 . 8 \% )}$ & \\
\hline
\end{tabular}

\section{Discussion}

SARS Cov-2 virus infection is a new disease worldwide. Though the virus primarily affects respiratory tract, there are several reports on various neurological presentation associated with COVID-19 worldwide $^{7-9}$. This small crosssectional study represents the pattern of stroke in a stroke unit of a tertiary care hospital. We found around almost equal number of patients in both sex. Their age ranging from 26 to 90 years where $58.5 \%$ patients were aged 60 and above. Study from China and Iranreported similar age of COVID-19 patients presenting with symptoms of stroke $^{10-12}$. Old age is an independent risk factor for stroke. Some case series also demonstrated stroke in relatively younger COVID-19 patients ${ }^{14}$. In this study around one fifth $(21.9 \%)$ of the patients are relatively younger $(<50$ years). Majority $(58.5 \%)$ of our patients came from different urban areas which may be due to the fact that the initial spread of the virus was more in the urban region. Hypertension $(68.3 \%)$ and diabetes (39\%) were the most common co-morbidities among the patients. Similar findings were observed in other studies $^{7,12,13}$.

Common presentation among the stroke patients were hemiplegia $(48.8 \%)$, altered level of consciousness (39\%), headache (12.2\%), convulsion (7.3\%). Hussain et $\mathrm{al}^{16}$ from Bangladesh showed almost same clinical presentation among the neurological COVID-19 patients. CT brain imaging revealed infarct in 39\% and hemorrhage in 51.2\%. Asadi-Pooya and Simani ${ }^{15}$ reported acute ischemic stroke in 5\% and acute hemorrhagic stroke in $0.5 \%$ of the patients in their systematic review of central nervous system manifestation of COVID 19 patients. Chest radiography by plain X-ray revealed opacities in the lungs in $17.1 \%$ cases in this study. The sensitivity of CT chest is obviously much higher. Ntaios et $\mathrm{al}^{17}$ found opacities in the lungs in $89.3 \%$ chest CT. The mortality rate in this study was $48.8 \%$ which is much higher than those reported in other study $(27.6 \%)^{17}$. This higher rate is probably due to the inclusion of both types of stroke (infarct and hemorrhage) patients. Death was relatively higher in hemorrhagic stroke in comparison to ischemic stroke and subarachnoid hemorrhage (SAH) but that was not statistically significant. It may be due to the sample size was comparatively small.

There is some limitation of this study. It is a single centre small cross-sectional study. Whether SARSCov-2 virus was a causative agent for stroke or it was just a co-infection in this pandemic situation is not certain as brain autopsy was not done in any patient. It was not possible to establish whether Stroke and COVID 19 was a mere coincidence or there had been a causal relationship.

\section{Conclusion}

In conclusion hemorrhagic stroke was the most commonly occurring stroke among the COVID-19 patients and associated with poor outcome. Whether SARSCov-2 virus was a causative agent for stroke or it just a co-infection in this pandemic situation is not certain. Therefore, the exact pathophysiology behind the stroke is still clearly unknown. Further large scalemulticenter study is warranted.

\section{References}

1. Zhu N, Zhang D, Wang W, Li X, Yang B, Song J, et al. A novel coronavirus from patients with pneumonia in China, 2019. N Engl J Med 2020; 382:727-733

2. World Health Organization (WHO). Rolling updates on 
coronavirus diseases (COVID-19). www.who.int/emergencies/ diseases/novel-coronavirus-2019/events-asthey-happen (accessed 13 April 2020)

3. Bohmwald K, Galvez N, Ríos M, Kalergis AM. Neurologic alterations due to respiratory virus infections. Frontiers in cellular neuroscience. 2018;12:386.

4. Baig AM, Khaleeq A, Ali U, Syeda H. Evidence of the COVID-19 virus targeting the CNS: tissue distribution, host virus interaction, and proposed neurotropic mechanisms. ACS Chemical Neuroscience. 2020 Apr 1;11(7):995-8.

5. Ellul MA, Benjamin L, Singh B, Lant S, Michael BD, Easton A, et al. Neurological associations of COVID-19. Lancet Neurol 2020; 19: 767-83

6. Varatharaj A, Thomas N, Ellul M, et al. UK-wide surveillance of neurological and neuropsychiatric complications of COVID-19: the first 153 patients. Lancet Psychiatry 2020; 7: 875-82

7. Li Y, Wang M, Zhou Y, et al. Acute cerebrovascular disease following COVID- 19: a single center, retrospective, observational study. Stroke Vasc Neurol. 2020 Sep; 5(3): 279-284

8. Helms J, Kremer S, Merdji H, Clere-Jehl R, Schenck M, Kummerlen C, et al. Neurologic features in severe SARS-CoV-2 infection. New England Journal of Medicine. 2020 Apr 15.

9. Chen T, Wu D, Chen H, Yan W, Yang D, Chen G, et al. Clinical characteristics of 113 deceased patients with coronavirus disease 2019: retrospective study. BMJ. 2020 Mar 26; 368.

10. Mao L, Jin H, Wang M, Hu Y, Chen S, He Q, et al. Neurologic manifestations of hospitalized patients with coronavirus disease
2019 in Wuhan, China. JAMA Neurol. 2020;77(6):683-90.

11. Pan Zhai, Yanbing Ding, Yiming Li et al. The impact of COVID-19 on ischemic stroke: A case report, 31 March 2020, PREPRINT (Version 1) available at Research Square

12. Karimi N, Rouhani N. COVID 19 and intra cerebral hemorrhage: causative or coincidental. New Microbes New Infect. 2020:100669

13. Haddadi K, Ghasemian R, Shafizad M. Basal Ganglia Involvement and Altered Mental Status: A Unique Neurological Manifestation of Coronavirus Disease 2019. Cureus. 2020;12(4):e7869

14. Oxley TJ, Mocco J, Majidi S, Kellner CP, Shoirah H, Singh IP, et al. Large-vessel stroke as a presenting feature of Covid-19 in the young. N Eng J Med. 2020;382(20):e60.

15. Asadi-Pooya AA, Simani L. Central nervous system manifestations of COVID-19: A systematic review. Journal of the Neurological Sciences. 2020 Apr 11:116832.

16. Hussain ME, Hoque MA, Alam MB, Yusuf MA, Chowdhury RN, Mohammad QD. Neurological Manifestations of COVID-19 patients: An Updated Review and Observations of COVID Patients in the National Institute of Neurosciences and Hospital, Dhaka, Bangladesh. Journal of Bangladesh College of Physicians and Surgeons. 2020;38:122-32.

17. Ntaios G, Michel P, Georgiopoulos G, Guo Y, Li W, Xiong, J, et al. Characteristics and Outcomes in Patients With COVID-19 and Acute Ischemic Stroke: The Global COVID-19, Stroke Registry.Stroke. 2020; 51(9):e254-e258 